\title{
Tenofovir Alafenamide Fumarate Induced Hypoglycemia in HIV Positive Patient with Insulin Dependent Diabetes
}

\author{
Taylor Donovan* \\ Seattle Infectious Disease Clinic, Washington, USA
}

\begin{abstract}
With the advances in highly active antiretroviral therapy, HIV is now considered a chronic disease. However, as the HIV population ages and have more co-morbidities, clinicians need to be increasingly aware about medication interactions. From our literature search, this is the first known case report of a patient switched from tenofovir disoproxil to tenofovir alafenamide and experienced improved renal proximal tubular function which we hypothesize led to improved insulin reuptake resulting in hypoglycemic episodes and subsequent need for insulin dose adjusting. Clinicians should be mindful of the potential for tenofovir alafenamide and insulin interactions and the need for vigilance when treating patients on high active antiretroviral therapy.
\end{abstract}

Keywords

Tenofovir alafenamide, Tenofovir disoproxil, Insulin, HIV, Proximal tubule

\section{Introduction}

Tenofovir disoproxil fumarate (TDF) is one of the most widely used and recommended medications used to treat HIV [1]. It is pro-drug of tenofovir diphosphate which acts as DNA analogue and halts the ability of reverse transcription to successfully create HIV viral DNA [2]. Since 2001, TDF has been considered and recommended as a backbone of modern day antiretroviral therapy. It has also been well documented that TDF causes damage to the renal proximal tubules through mitochondrial toxicity and ATP shortage in the tubular cells leading to proximal tubular dysfunction [3]. Consequently, TDF is associated with improper reabsorption of ions and small molecules from the urate at the proximal tubule [4]. Bone mineral density loss and renal toxicity is associated with TDF. A newer tenofovir diphosphate pro-drug, tenofovir alafenamide fumarate (TAF) was introduced and approved in 2015 and is also a pro-drug of tenofovir diphosphate. But TAF is associated with lower serum levels than TDF and higher intracellular levels than TDF, thus reducing the risk of developing renal and bone density issues [5].

Animal studies have show metabolism of Humalog to be identical to that of human insulin and can be influenced by renal impairment [6]. However, urine protein/creatine ratio, and glycosuria are rough markers of proximal tubular dysfunction so it is plausible that the patient had significant proximal tubular dysfunction [4]. The proximal tubular dysfunction induced by TDF has also been shown to reversible with cessation of TDF therapy.

The available laboratory results only suggest possible proximal tubular dysfunction.

\section{Case Presentation}

A 51-year-old man with HIV and type 1 diabetes, being treated with darunavir/cobicistat with tenofovir disoproxil fumarate/emtricitabine and a Humalog insulin pump with stable dosing and an $\mathrm{HbA1C}$ of 9.3. His insulin dose had been unchanged by his endocrinologist for the prior four years and was 1.15 units per hour. Other comorbidities include hypertension and chronic kidney disease, hypogonadism, depression, erectile dysfunction. On 4/28/16 his urine protein/creatinine ratio was $218 \mathrm{mg} / \mathrm{g}$, serum glucose was $183 \mathrm{mg} / \mathrm{dl}$, urine glucose of $3+$ on urinalysis, $\mathrm{HbA1C}$ of $9.3 \%$, and serum creatinine was $0.85 \mathrm{mg} /$ dl. He was switched from tenofovir disoproxil fumarate (TDF) to tenofovir alafenamide fumarate (TAF) in the setting of viral suppression in October of 2016. 2.5 weeks after switching TDF to TAF he presented to the clinic with a history of experiencing two separate hypoglycemic episodes at $39 \mathrm{mg} / \mathrm{dl}$ and $41 \mathrm{mg} / \mathrm{dl}$ and altered mental status changes which were successfully at home with oral glucose therapy. No obvious precipitating cause was found in the history. His Humalog was reduced to 0.85 units per hour and the hypoglycemic episodes subsided.

*Corresponding author: Taylor Donovan, MSN, ARNP Seattle Infectious Disease Clinic 509 Olive Way, Ste 752, Seattle, WA, 98101, USA

Accepted: January 17, 2019

Published online: January 19, 2019

Citation: Donovan T (2019) Tenofovir Alafenamide Fumarate Induced Hypoglycemia in HIV Positive Patient with Insulin Dependent Diabetes. Clin J HIV AIDS 3(1):36-37 
On 3/15/2017 his urine protein/creatinine ratio was 122 $\mathrm{mg} / \mathrm{g}$, his serum glucose was $205 \mathrm{mg} / \mathrm{dl}$, urine glucose of $3+$ on urinalysis, $\mathrm{HbA} 1 \mathrm{C}$ was $9.8 \%$, and creatinine was $0.95 \mathrm{mg} /$ dl.

This possibly represents a case of improved proximal tubular function after switching from TDF to TAF causing increased Humalog reabsorption at the proximal tubule and thus a longer half-life triggering hypoglycemic episodes.

\section{Discussion}

Prescribers should be aware of patients regaining proximal tubular function in patients on insulin therapy as this may lead to prolonged insulin half lives and hypoglycemic episodes.

\section{References}

1. Herlitz LC, Mohan S, Stokes MB (2010) Tenofovir nephrotoxicity: Acute tubular necrosis with distinctive clinical, pathological, and mitochondrial abnormalities. Kidney Int 78: 1171-1177.

2. Cote HC, Magil AB, Harris M, et al. (2006) Exploring mitochondrial nephrotoxicity as a potential mechanism of kidney dysfunction among HIV-infected patients on highly active antiretroviral therapy. Antivir Ther 11: 79-86.

3. Tourret J, Deray G, Isnard-Bagnis C (2014) Tenofovir effect on the kidneys of HIV-infected patients: A double-edged sword? J Am Soc Nephrol 24: 1519-1527.

4. Hall AM, Bass P, Unwin RJ (2014) Drug-induced renal Fanconi syndrome. QJM 107: 261-269.

5. De Clercq E (2016) Tenofovir alafenamide (TAF) as the successor of tenofovir disoproxil fumarate (TDF). Biochem Pharmacol 119: 1-7.

6. (2017) Humalog Package Insert [Pamphlet] Eli Lilly and Company, Indianapolis.

DOI: $10.36959 / 695 / 566$

Copyright: (C) 2019 Donovan T. This is an open-access article distributed under the terms of the Creative Commons Attribution License, which permits unrestricted use, distribution, and reproduction in any medium, provided the original author and source are credited. 